\title{
Apomorphin und Psychostimulantiensucht
}

\section{Apomorphine and Psychostimulant Addiction}

\author{
Juan D. Delius, Sabine Keller und Martin J. Acerbo
}

Die Menschen können nicht sagen, wie sich eine Sache zugetragen, sondern nur, wie sie meinen, dass sie sich zugetragen hätte

(Georg C. Lichtenberg, 1773, Sudelbücher).

\section{Einleitung}

Apomorphin wurde Mitte des 19. Jahrhunderts ausgehend von Morphium hergestellt. Gleich nach der Synthese fiel dessen emetische Wirkung auf. Eine analgetische und euphorisierende Wirkung wie Morphium hatte es nicht. Klinisch ist es dann in höheren Dosen bei Vergiftungen und später auch zeitweise bei der Alkoholaversionstherapie verwendet worden. Tierversuche haben aber dann gezeigt, dass es in niedrigeren Dosen auffällige orale Stereotypien auslöst. Bei Kröten und Fischen sind das Schnappanfälle, bei Tauben und Hühnern Putz- und Pickanfälle und bei Ratten und Mäusen Kau- und Nageanfälle. Auch bei Menschen werden u. U. Kaustereotypien beobachtet. In den 50er Jahren wurden bei Ratten und Mäusen Ähnlichkeiten mit der Wirkung von Psychostimulanzien, insbesondere Amphetamin und Kokain, erkannt. Labortechnisch äußert sich das nicht zuletzt dadurch, dass es nach Gaben dieser Drogen und Apomorphin bei den Nagern neben den Stereotypien zu nachhaltigen, leicht messbaren Laufaktivitätssteigerungen in Aktometern kommt. Dadurch, dass Amphetamin und Kokain als Dopaminagonisten erkannt wurden, unter anderem weil sie bei wiederholter Einnahme zu psychoseähnlichen Symptomen führten und Schizophrenie wiederum als eine Hyperdopaminie angesehen wurde, kam es zur Feststellung, dass Apomorphin ebenfalls ein Dopaminagonist sei. Der beste Beweis dafür ist, dass Haloperidol, der klassische Dopaminantagonist, sehr effizient die pharmakologische Wirksamkeit von Apomorphin zu unterbinden vermag. Inzwischen ist es nachgewiesen, dass sich Apomorphin direkt und spezifisch an die Dopaminrezeptoren der D1 und D2 Familien bindet und dies mit einer Effektivität, die die des Transmitters Dopamin um etwa das Tausendfache übersteigt. Das ist auch ein Grund, warum Apomorphin, allerdings meistens nur vorïbergehend, in der Parkinsontherapie verwendet wird. Neuerdings soll es in niedrigen Dosen als männliches Sexualstimulantium, welches vielleicht Viagra ausstechen kann, vermarktet werden.

Kokain und Amphetamin sind im Gegensatz zu Apomorphin indirekte und nicht ganz so spezifische Agonisten des Dopamins, wobei die erste Substanz vornehmlich den Rücktransport des Transmitters aus dem synaptischen Spalt in die präsynaptischen Endigungen blockiert und die zweite Substanz vornehmlich eine vermehrte Ausschüttung von Dopamin aus den präsynaptischen Endigungen fördert. Inwieweit die direkten sehr spezifischen und die indirekten eher unspezifischen Wirkungsweisen der Drogen damit zu tun haben, dass Apomorphin gar nicht, aber Amphetamin und Kokain suchtauslösend wirken, wird noch $\mathrm{zu}$ kommentieren sein.

\section{Belohnungssystem}

Es ist wohl bekannt, dass Ratten bereit sind, einen geeigneten Schalter wiederholt zu bedienen, um sich dadurch jeweils eine kurz dauernde elektrische Reizung verschiedener Gehirngebiete zu verschaffen. Die Orte, die solches Hirnselbstreizungsverhalten aufrechthalten, sind weit verstreut im Mittel-, Zwischen- und Endhirn lokalisiert. Es ist nach und nach deutlich geworden, dass sich dieses so genannte Belohnungssystem weitgehendst mit einem mestelencephalen Projektionssystem deckt, das sich des Transmitters Dopamin bedient. Die dopaminergen Axone von Zellkörpern, die in der Substantia nigra und im Tegmentum ventrale liegen, ziehen durch das Zwischenhirn und endigen im Vorderhirn, dort bevorzugt, aber nicht nur, im Striatum und im präfrontalen Cortex. Passend dazu hat sich dann auch gezeigt, dass die systematische aber auch geeignet lokale Verabreichung von Dopaminagonisten (darunter Amphetamin, Kokain und Apomorphin) oder -antagonisten (darunter Haloperidol) das Selbstreizungsverhalten 
fördert, beziehungsweise hemmt (Gardner, 1997). Dass der Begriff Belohnungssystem angemessen ist, wird nicht nur dadurch belegt, dass die Hirnreizung im Rahmen des instrumentellen Lernens analog wie konventionelle Belohnungen (Futter, Wasser, usw.) wirkt, sondern auch dadurch, dass solche konventionellen Belohnungen genau wie die Selbsthirnreizung, zur Ausschüttung von Dopamin mindestens im ventralen Striatum führt. Nicht zu vergessen ist allerdings der Umstand, dass das mes-telenzephale dopaminerge Projektionssystem auch das übrige Striatum innerviert und dort motorische Reaktionen aktivieren kann, zu denen u. a. auch die anfänglich erwähnte Laufaktivität und Stereotypien gehören, die von den psychostimulatorischen Drogen ausgelöst werden können.

Aus theoretischer Sicht ist eine weit verzweigte neurale Bahn wie die beschriebene zu erwarten, um die verschiedenartigsten Verknüpfungen, die beim belohnten sensomotorischen Lernen möglich sind, hervorzubringen. Dass diese Verknüpfungen mittels glutamato-dopaminergen Synapsensystemen im ventralen Striatum zustandekommen könnten, wurde zunächst aufgrund von histologischen Befunden vermutet. Die Vorstellung, dass dem Verknüpfungsvorgang eine dopamingeförderte langzeitpotenzierungsartige Effizienzsteigerung von glutamatergen Synapsen der cortico-striatalen Bahnen zugrunde liegen könnte, hat in letzter Zeit vielfache empirische Unterstützung bekommen (Kelley, 1999). Wie dem auch sei, das dopaminerge Belohnungssystem kommt in weitgehendster homologer Ausformung bei allen Wirbeltieren vor, Menschen und Tauben eingeschlossen. Durch einige Patientenberichte, die bei Hirnselbstreizungsversuchen im Rahmen von explorativen Vorbereitungen zu Hirnchirurgien anfielen, ist belegt, dass die Populärbezeichnung »Pläsiersystem« für das Belohnungssystem nicht ganz unzutreffend ist. Mindestens mittelbar hat es mit der Auslösung von positiven Emotionen zu tun. Viele verschiedenartige Befunde, aber nicht zuletzt ein Ansteigen des Dopaminpegels im ventralen Striatum belegen, dass die Einnahme vieler, wenn nicht aller abhängigkeitsverursachenden Drogen, wenn nicht direkt dann doch indirekt, dieses Belohnungssystem pharmakologisch aktiviert. Sie werden ja auch mindestens teilweise konsumiert, weil sie einen subjektiven Pläsier- oder sogar Euphorie-Effekt hervorrufen. Es existiert natürlich auch ein Bestrafungssystem, das sich antagonistisch zum Belohnungssystem verhält, über das aber aus Tierschutzgründen sehr viel weniger bekannt ist.

\section{Hypersensitivierung}

Die Sucht nach Kokain und Amphetamin, wie auch nach vielen anderen Drogen, stellt sich erst nach einem mehrfach wiederholten Drogenkonsum ein. Somit ist davon auszugehen, dass eine mehr oder minder autogen auftretende Suszeptibilitätsänderung bei der Suchtbildung eine Rolle spielt. Allerdings beginnen wir hier mit einer Drogenwirksamkeitsänderung, der Hypersensibilisierung, die am deutlichsten durch einen radikalen Fremdeingriff zustandekommt. Diese Empfindlichkeitssteigerung tritt beim dopaminergen System am deutlichsten bei chirurgischer oder chemischer Denervierung auf. Lädiert man die Substantia nigra einseitig mittels Hochfrequenzkoagulation oder toxischer 6-Hydroxydopaminapplikation, kommt es bei Ratten gleich nach dem Eingriff zu ipsiversiven lokomotorischen Drehtendenzen dadurch, dass es mit dem Ausfall der entsprechenden nigro-striatalen Bahn $\mathrm{zu}$ einem präsynaptischen Dopaminausscheidungsdefizit im ipsilateralen Striatum kommt. Im Laufe der Zeit schwächt sich das Drehsyndrom bis zur praktisch völligen Remission ab. Systemische Gaben von Dopaminagonisten vermögen das anfängliche ipsiversive Drehen teilweise zu unterdrücken; aber später, wenn die Tiere als spontan kompensiert gelten, lösen dieselben Drogengaben starke kontraversive Drehtendenzen aus. Diese Befunde erklären sich dadurch, dass es wegen des Dopamindefizits bei den ipsilateralen postsynaptischen Striatumneuronen zu einer Aufwärtsregulation der Dopaminempfindlichkeit kommt. Dies dadurch, dass es zu einer örtlichen Steigerung der Zahl von Dopaminrezeptoren und/oder deren Dopaminaffinität kommt. Eine globalere aber ansonsten ähnlich verursachte Hypersensibilität lässt sich bei Ratten durch eine länger dauernde Verabreichung von Dopaminantagonisten, wie Haloperidol auslösen. Anschließend verursacht der Agonist Apomorphin, aber auch Amphetamin und Kokain, bei den so vorbehandelten Tieren eine im Vergleich mit Kontrolltieren deutlich erhöhte motorische Aktivität, die vermutlich auf die vorausgehende kompensatorische Steigerung der Dopaminrezeptivität zurückzuführen ist. 


\section{Toleranz}

Wenn man die Erscheinung der Denervations- bzw. der antagonistenverursachten Hypersensibilisierung als einen homeostatischen Regelungsvorgang interpretiert, kann man das Vorkommen eines entsprechenden regulatorischen Gegeneffektes erwarten. Nämlich dass es bei einem Überangebot an Transmittern, oder bei chronischen Gaben eines Agonisten, zu einer kompensatorischen Abregulierung der postsynaptischen Empfindlichkeit über eine Reduktion der Rezeptorendichte bzw. deren Affinität kommt. Unter besonderen Umständen ist allerdings hierbei aúch mit einer Kompensation über eine präsynaptische Verminderung der Transmitterausscheidung zu rechnen. Eine Empfindlichkeitsminderung bei wiederholter Gabe von Psychopharmaka mit transmitteragonistischen Eigenschaften ist tatsächlich ein verbreitetes Phänomen. Unter den suchtverursachenden Drogen ist eine solche Toleranzentwicklung bei Morphium und dessen Abkömmlingen wohl bekannt. In dem Maße, in welchem Suchtdrogen als direkte oder auch indirekte Agonisten von Transmittern wirksam sind, muss bei Toleranz zunächst mal als eine wie auch immer geartete homeostatische Regulierung der synaptischen Wirksamkeit vermutet werden. Ein Zuviel an synaptischer Aktivierung sollte zu einer Dämpfung der synaptischen Übertragung führen. $\mathrm{Ob}$ allerdings Drogentoleranz regelmäßig mit solchen verhältnismäßig unkomplizierten kompensatorischen synaptischen Veränderungen verbunden ist, ist nicht allgemein belegt.

Eher besser belegt ist, dass es im Zusammenhang mit der Toleranzbildung häufig zu einer konditionierten Toleranzreaktion kommt, in dem Sinne, dass der Grad der Toleranz, die bei einer akuten Drogengabe wirksam wird, davon abhängig ist, inwieweit die umgebende Reizsituation bei dieser Gabe jener gleicht oder mindestens ähnelt, die bei den vorausgehenden toleranzverursachenden Drogeneinnahmen vorherrschte (Kim, Siegel und Patenall, 1999). Sind die Umgebungsbedingungen bei solchen PosttoleranzGaben hinreichend verschieden, dann ist in der Regel die Toleranz gegenüber der Droge von leicht bis stark gemindert. Dieser Effekt ist besonders im Zusammenhang mit Lethalwirkungen von Drogen bekannt geworden, aber er dehnt sich wohl auch auf das Aus$\mathrm{ma} ß$ der Pläsierwirkungen von toleranzanfälligen Drogen aus. Wichtig ist in diesem Zusammenhang, dass man bei der konditionierten Toleranz wohl eher mit primären Veränderungen bei den glutamatergen Synapsen, als mit Veränderungen bei den Synapsen, auf welche die Droge direkter wirken, rechnen muss. Und zwar deswegen, weil Lernvorgänge nach den derzeitigen Erkenntnissen mit der besonderen Plastizität von Synapsen, die sich des Transmitters Glutamat bedienen, zusammenhängen.

Das wohl einfachste Modell zur Suchtentwicklung ergibt sich daraus, dass der Belohnungseffekt bzw. die Pläsierreaktion auf Drogen einer wie eben beschriebenen Toleranzentwicklung anheim fällt, die nur mit entsprechend erhöhten Drogendosen überwunden werden kann. Der Drogenentzug führt dazu, dass die Verstärkungslage bzw. der hedonistische $\mathrm{Zu}$ stand in den bestrafenden-aversiven Bereich abgleitet, der im vortoleranten Stadium nur durch das Auftreten von explizit strafenden-aversiven Reizkonstellationen zustande kommen konnte, nicht aber durch schlichte Drogenabwesenheit (Koob und Bloom, 1988).

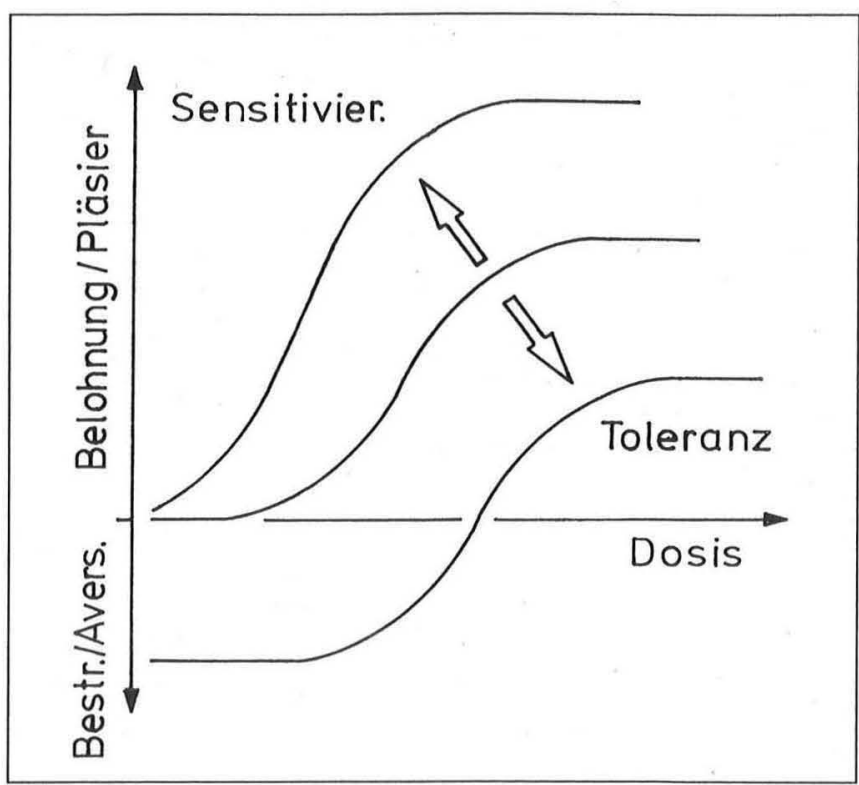

Abbildung 1: Angenommene Verschiebungen der Drogenwirksamkeit auf der Belohnungs-/Bestrafungsdimension bzw. der Pläsier-Aversionsdimension (schematisch)

\section{Sensitivierung}

Das Toleranzmodell der Sucht dürfte bei Drogen gelten, deren wiederholte Einnahme in der Regel tatsächlich zu ausgeprägten Toleranzerscheinungen führt, wie sie z. B. bei Opioiden und Alkohol grob zutreffen. Nicht passend ist das Modell für die Suchtdrogen, bei denen eine wiederholte Einnahme vorherrschend $\mathrm{zu}$ einer Sensitivierung führt (Abbildung 1). Das gilt insbesondere für suchtgenerierende 
Psychostimulanzien wie Amphetamin und Kokain. Wir haben uns intensiv mit der Sensitivierung auf das allerdings nicht suchtverursachende Apomorphin beschäftigt. Wie bereits erwähnt, löst das Apomorphin bei Tauben in einer Dosis von $0,5 \mathrm{mg} / \mathrm{kg}$ intramuskulär einen Pickanfall aus. Das ist zunächst ein reiner motorischer Effekt, der aber eine sehr deutliche Steigerung durch Sensitivierung erfährt. Auf die erste Injektion hin sind es einige Hunderte von Pickern. Nach sechs Injektionen in Tagesabständen steigert sich der Anfall, der von derselben Dosis Apomorphin ausgelöst wird, auf mehrere Tausend Picker und er dauert jetzt länger als eine Stunde. Wichtig ist der Umstand, dass diese Sensitivierung sehr lange erhalten bleibt. Erst nach rund zwei Jahren fällt die Pickhäufigkeit, die diese Standarddosis auslöst, auf die Hälfte derjenigen, die gerade nach der abgeschlossenen Sensitivierung gemessen wurde. Aber auch dann läuft eine Wiedersensitivierung noch deutlich beschleunigt $a b$.

Es fiel uns auf, dass Tauben, die sensitiviert waren, auch wenn sie unbehandelt in den mit Apomorphinbehandlung verbundenen Versuchkäfig gesetzt wurden, Pickschübe zeigten, die sie in andersartigen Kontrollkäfigen, in denen sie mit Saline-Injektionen behandelt worden waren, nicht zeigten. Wir konnten beweisen, dass diese Reaktion eine konditionierte Reaktion nach Pawlow darstellt. Weiterhin konnten wir zeigen, dass der Pickreaktionszuwachs, der bei der Sensitivierung auftritt, im Wesentlichen durch die fortlaufende Addierung einer durch die Käfigumgebung bedingten konditionierten Reaktion mit einer durch das Apomorphin ausgelösten unkonditionierten Reaktion zustande kommt. Wird die Sensitivierungsbehandlung in einer Serie verschiedener Käfige durchgeführt, dann bleibt die Reaktionssteigerung vernachlässigbar gering. Dadurch wird die ausgesprochene Umgebungsabhängigkeit der Sensitivierrung belegt, wie das nach der Konditionierungshypothese zu erwarten ist. Dieser Befund steht wiederum im Einklang mit der Vermutung, dass Apomorphin, wie es für einen potenten Dopaminagonisten zu erwarten ist, einen deutlichen Belohnungseffekt hat. Tauben bevorzugen, wenn sie die Wahl haben, einen Käfig, in dem sie zuvor mit der Droge behandelt worden sind, vor einem, in dem sie mit Saline behandelt wurden. Bei Säugern ist darüber hinaus gezeigt worden, dass Apomorphin ein stetiges Selbstinjektionsverhalten aufrechthalten kann. Weniger übereinstimmend mit der Annahme, dass die Sensitivierung durch einen Lernvorgang zustandekommt, ist die Beobachtung, dass weder eine Vorhemmungs- oder eine Löschungsbehandlung (mehrfach wiederholte Nur-
Saline-Behandlung im Versuchskäfig entweder vor oder nach dem Sensitivierungstraining) so gut wie keine Schwächung des Sensitivierungseffektes hervorruft. Darüber hinaus entspricht die nach der Sensitivierung beobachtete konditionierte Pickreaktion von seiner quantitativen Ausprägung nur teilweise dem Pickreaktionszuwachs, der sich im Laufe der Sensitivierung entwickelt. Das allerdings mag damit zu tun haben, dass es wohl falsch ist, Apomorphingaben nur mit einem unkonditionierten Reiz gleichzusetzen. Der Umstand, dass es in der Retina Dopaminrezeptoren gibt und dass deren Aktivierung die Wahrnehmung beeinflusst, als auch dass Tauben beim Drogenunterscheidungslernen nachweislich einen Apomorphinzustand interozeptiv von einem Salinezustand unterscheiden können, zeigt dass Apomorphin z. T. auch die Eigenschaften eines konditionalen Reizes haben kann. Das sollte zu einer Autokonditionierung führen, die im äußerlichen Verlauf der Sensitivierung entspricht. Man kann aber diesem Vorgang alleine nicht dem gesamten Sensitivierungseffekt zuschreiben. Externe konditionierte Reize spielen schon eine wesentliche Rolle. Tauben, die z. B. in einem weißwandigen grünbepunkteten Käfig auf Apomorphin sensitiviert wurden und parallel in einem schwarzwandigen, rotbepunkteten Käfig mit Saline behandelt wurden, bepickten anschließend bei Wahlmöglichkeit sowohl unter Apomorphin als auch unter Saline, fast ausnahmslos nur das erste, apomorphinassoziierte Wanddekor.

\section{Neuropharmakologie}

Eine Doppelbehandlung mit Apomorphin und Haloperidol verhindert die Entwicklung der auf die Pickreaktion bezogenen Sensitivierung völlig. Es besteht also keine Frage, dass für das Zustandekommen der Sensitivierung eine Aktivierung von Dopaminrezeptoren essenziell ist. Wenn aber erst die Sensitivierung durch Training mit nur Apomorphin sich entwickelt hat, dann hat eine Haloperidolbehandlung keine Wirkung mehr. Das heißt, die Akquisition aber nicht die Expression der konditionierten Reaktion kann von einem Dopaminantagonisten unterdrückt werden. Anders ist es bei dem Glutamatantagonisten MK 801. Er vermag sowohl die Akquisition als auch die Expression der konditionierten Reaktion zu hemmen. Dies lässt sich mit Befunden vereinbaren, dass im Striatum, besonders im ventralen Striatum und vielleicht noch genauer im Nucleus accumbens, die Effektivität von glutamatergen Synapsen der corticostriatalen, reizsignalisierenden Bahnen durch kon- 
tingente Aktivierung der dopaminergen Synapsen der mesolimbischen, belohnüngssignalisierenden Bahn gesteigert wird, Die angesprochene synaptische Effektivitätsänderung entspräche einer dopaminerg geförderten Langzeitpotenzierung bzw. einer Langzeitdepression, wie sie inzwischen bei striatalen Dickschnitten physiologisch/pharmakologisch vorkommend hinreichend nachgewiesen worden sind. Die Abbildung 2 stellt schematisch vereinfacht die neuronalen Grundlagen der Sensitivierung bzw. der ihr zugrunde liegenden konditionierten Reaktion zusammen. Neuerlich haben wir übrigens in unserer Taubenzucht eine Verwandtschaft entdeckt, die apomorphinunempfindliche Nachkommen zeugt. Von ihnen erhoffen wir uns weiterführende Erkenntnisse.

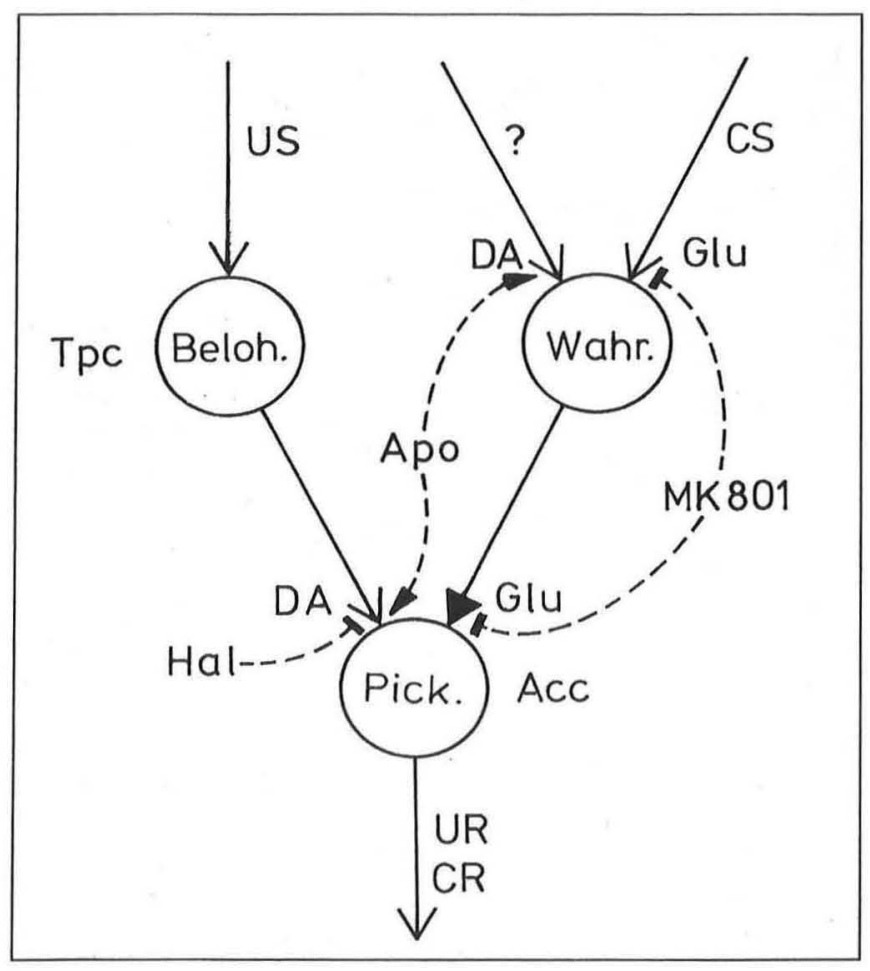

Abbildung 2: Modell zur Apomorphinwirkung bei Tauben. Abkürzungen: unkonditionierte Stimuli, US; Belohnungssystem, Beloh.; dopaminerge Synapsen, DA; Substantia nigra/Tegmentum ventrale Homologon bei Vögeln, Tpc; Apomorphin, Apo; pickmotorisches Neuron, Pick.; unkonditioniertes Picken, UR; konditionierte Stimuli, CS; glutamaterge Synapsen, Glu; Wahrnehmungsneuron, Wahr; konditionierte Reaktion CR; DAntagonist Haloperidol, Hal; Gluantagonist, MK801. Die ausgefüllte Glusynapse gilt als konditionierungsbedingt veränderlich.

\section{Sucht}

Ergebnisse wie die, welche gerade zur Sensitivierung der pickauslösenden Wirkung von Apomorphin berichtet wurden, ließen sich, wenn auch vielleicht nicht so geradlinig, für die Sensitivierung der motorisch aktivierenden Wirkung von Amphetamin, Kokain und auch Apomorphin bei Ratten zusammenstellen. Es stellt sich die Frage, wieso Amphetamin und Kokain, bei denen eine wiederholte Einnahme vorherrschend zu einer Sensitivierung anstatt zu einer Toleranz führen, überhaupt zu Suchterscheinungen führen. Es ist überdeutlich, dass bei den beiden Drogen das vorher skizzierte Modell, das auf einer Toleranzentwicklung aufbaut, kaum zutreffen kann. Vorstellbar ist, dass in diesem Falle es durch wiederholte Drogeneinnahme neben der Sensitivierung der motorischen Aktivierung zu einer parallelen progressiven Steigerung des motivational wirksamen Anreizwertes der Droge kommt. Es entsteht ein gesteigertes psychologisch-neurales Verlangen, weniger ein physiologisch-vegetatives Bedürfnis für eine erneute Drogeneinnahme. Dies mag mit der langfristigen Gedächtnisspeicherung einer überzogenen Erwartung bezüglich der pläsierauslösenden Wirkung der Drogen gleichzusetzen sein und könnte mit dem Entstehen eines subjektiven Lechzen nach der Droge zusammenhängen (Robinson und Berridge, 1993).

Warum aber darüber hinaus die Dopaminagonisten Amphetamin und Kokain jedoch nicht der Dopaminagonist Apomorphin suchtgenerierend wirkt, ist noch weitgehend unklar. Der Fakt, dass Apomorphin auch eine emetische, also aversive Wirkung haben kann, ist wohl kaum maßgeblich weil diese Wirkung erst bei verhältnismäßig hohen Dosen auftritt. Möglicherweise liegt es daran, dass Apomorphin eine direktere Wirkung auf postsynaptische Rezeptoren hat und ein sehr spezifischer dopaminerger Agonist ist, während Amphetamin und Kokain nur indirekte, durch den präsynaptischen Dopamintransporter wirkende Dopaminagonisten sind, die zumal unspezifisch, wenn auch schwächer als Serotonin- und Noradrenalinagonisten wirken. Vielleicht ist es gerade diese Mehrfachwirksamkeit, die über die Sensitivierung bei motorischen Reaktionen hinaus auch zu der Sensitivierung des Drogenbedürfnisses bei Amphetamin und Kokain führt. Übrigens, ob anders als bei Säugern, sich bei Tauben und überhaupt Vögeln, eine Sucht auf Psychostimulanzien entwickeln kann, ist ebenfalls eine noch offene Frage. 


\section{Literatur}

Acerbo, M. J, Godoy, A. M. und Delius, J. D. (1999). Longterm retention of apomorphine conditioning in pigeons. In: N. Elsner und U. Eysel (eds.), Göttingen Neurobiology Report, p. 562. Thieme, Stuttgart.

Burg, B., Haase, C., Lindenblatt, U. und Delius, J. D. (1989). Sensitization to and conditioning with apomorphine in pigeons. Biochemistry, Pharmacology and Behavior, 34, 59-64.

Gardner, E. L. (1997). Brain reward mechanisms. In: Lowinson, J. H., Ruiz, P., Millman R. P. und Langrod, J. G. (Hrsg.) Substance Abuse: A Comprehensive Textbook. Williams and Wilkins, Baltimore.

Godoy, A. und Delius, J. D. (1999). Sensitisation to apomorphine in pigeons is due to conditioning, subject to generalization but resistant to extinction. Behavioural Pharmacology, 10, 367-378.

Godoy, A. M., Siemann, M. und Delius, J. D. (2000). Dose shift effects on an apomorphine induced response. Medical Science Research, 28, 39-42.

Keller, S. und Delius, J. D. (2001). Discriminative learning occasioned by the administration of a dopamine agonist. Psychopharmacology, im Druck.

Kelley, A. E. (1999). Neural integrative activities of nucleus accumbens subregions in relation to learning and motivation. Psychobiology, 27, 198-213.
Kim, J. A., Siegel S. und Patenall, V. R. A. (1999). Drugonset cues as signals: intraadministration associations and tolerance. Journal of Experimental Psychology: Animal Behaviour Processes, 25, 491-504.

Lindenblatt, U. und Delius, J. D. (1987). Apomorphineinduced pecking in pigeons classically conditioned to environmental cues. Psychopharmacology, 93, 223-225.

Robinson, T. E. und Berridge, K. C. (1993). The neural basis of drug craving. Brain Research Reviews, 18, 247-291.

Koob, G. F. und Bloom, F. E. (1988). Cellular and molecular mechanisms of drug dependence. Science, 242, 715-722.

Wynne, B. und Delius, J. D. (1995). Sensitization to apomorphine in pigeons: unaffected by latent inhibition but still due to classical conditioning. Psychopharmacology, $119,414-420$.

\section{Korrespondenzanschrift}

Prof. Dr. Juan D. Delius

Allgemeine Psychologie

Universität Konstanz

78457 Konstanz

E-Mail: juan.delius@uni-konstanz.de 